\title{
Dissipative area-preserving one-dimensional Fermi accelerator model
}

\author{
Edson D. Leonel \\ Departamento de Estatística, Matemática Aplicada e Computação, Instituto de Geociências e Ciências Exatas, Universidade Estadual \\ Paulista Av.24A, 1515, Bela Vista, CEP 13506-700, Rio Claro, São Paulo, Brazil \\ P. V. E. McClintock \\ Department of Physics, Lancaster University, Lancaster, LA1 4YB, United Kingdom \\ (Received 19 December 2005; revised manuscript received 8 May 2006; published 27 June 2006)
}

\begin{abstract}
The influence of dissipation on the simplified Fermi-Ulam accelerator model (SFUM) is investigated. The model is described in terms of a two-dimensional nonlinear mapping obtained from differential equations. It is shown that a dissipative SFUM possesses regions of phase space characterized by the property of area preservation.
\end{abstract}

DOI: 10.1103/PhysRevE.73.066223

PACS number(s): 05.45.Ac, 05.45.Pq, 47.52. $+\mathrm{j}$

\section{INTRODUCTION}

Following Enrico Fermi's seminal paper [1], the onedimensional Fermi accelerator model has been studied over many years, using a range of different approaches. The model was originally introduced as a possible mechanism by which cosmic rays can acquire their enormous energies. It involves acceleration of charged particles through their interaction with periodically varying magnetic fields. Fermi's model was subsequently modified in order to take into account many other physical situations. One variant is the wellknown Fermi-Ulam accelerator model [2-4] (FUM). It consists of a classical particle confined between two rigid walls, one fixed and the other moving in time, in the absence of external fields. For periodic motion of the moving wall, the main results can be summarized as: (i) at low energy, the phase space presents a chaotic sea that (ii) surrounds Kolmogorov-Arnol'd-Moser (KAM) islands, whereas at high energy (iii) there is a set of invariant spanning curves. Many observable, e.g., Lyapunov exponents, average velocity, roughness, etc., may be studied and characterized in the chaotic sea at low energy. For example, scaling arguments [5] can be used to describe the behavior of both average velocity and the corresponding deviation around it-the roughness (see also Ref. [6] for recent results in the simplified FUM). A different version that considers the presence of external fields, sometimes known as the bouncer [7], consists of a classical particle in the presence of a constant gravitational field, bouncing elastically against a rigid but periodically moving wall. Remarkably, for appropriate control parameters and initial conditions and in contradistinction to the FUM, it is possible to observe unlimited growth in the average velocity and energy of the particle. This apparent discrepancy was later explained by Lichtenberg, Lieberman, and Cohen [8] who showed that, although the models are very similar, there are intrinsic differences leading to different asymptotic behavior. Recently, a hybrid version of these two models has been proposed [9]. It consists of a classical particle confined to bounce elastically between two rigid walls, one fixed and the other moving periodically; in addition, there is a constant external field. For certain ranges of control parameters and appropriate initial conditions, the system possesses properties that are individually present in the FUM and bouncer models, but which coexist together in the hybrid version of the model.

There are also many other variants of the basic Fermi model considering, for example, the consequences of dissipation and even quantum effects [10-14]. Dissipation can be introduced, e.g., via consideration of inelastic collisions [15-21], a chaotic attractor may be present, regions of locking in the phase space may be observed, crisis events may be characterized [22], and the effect of a drag force [23,24] where transients and attracting fixed points are present can be investigated. The importance of studying these systems is that they allow direct comparison of theoretical results with experimental data [25-27]. In addition, the formalism and methods used in the description of these models are directly extendable to billiards problems [28].

In this paper, we revisit the FUM in order to study the case where the particle is immersed in a fluid and therefore suffers the action of a frictional (drag) force. We will consider the simplified version of the complete FUM (the SFUM): we thus assume that both walls are fixed but that, after the particle collides with one of them, it suffers a change of momentum as if the wall were moving. Following its introduction by Lieberman and Lichtenberg [3], this simplification of moving boundaries in mappings has been widely applied $[4,5,16,18,20,21,29]$. We have ourselves used a simplified version of the hybrid Fermi-Ulam-bouncer model [9] in order to obtain analytically the existence condition for invariant spanning curves in both the low and high energy domains.

The organization of the paper is as follows. In Sec. II we present the model and discuss all the details needed for the construction of the nonlinear mapping. Our numerical results are also presented and discussed in this section. Final remarks are made, and conclusions drawn, in Sec. III.

\section{THE MODEL AND NUMERICAL RESULTS}

The model in question thus consists of a confined classical particle of mass $m$ bouncing between two rigid walls in the presence of a drag force whose magnitude is assumed to be proportional to the particle's velocity, $F_{v}=-\eta^{\prime} v$, where $\eta^{\prime}$ is the viscosity. It is assumed that gravity and other external 
fields are absent. One wall is fixed at $x=l$ and the other one is located at the origin $x=0$. The collisions with both walls are assumed to be elastic but we assume that, after hitting the wall at $x=0$, the particle changes momentum as if the wall were moving according to the equation $x_{w}=\varepsilon \cos (\omega t)$. The dynamics is described by using a two-dimensional nonlinear mapping $T\left(v_{n}, t_{n}\right)=\left(v_{n+1}, t_{n+1}\right)$ for the velocity and corresponding time variables. Supposing that the initial conditions are $v=v_{n}, t=t_{n}$ with the particle's initial position at $x=0$, and after solving Newton's second law $-\eta^{\prime} v=m d v / d t$, we find that the velocity of the particle is given by $v_{p}(t)=v_{n} e^{-\eta t}$. Its corresponding position is $x_{p}(t)=v_{n} / \eta[1-\exp (-\eta t)]$. The variable $\eta$ is defined as $\eta=\eta^{\prime} / m$. Note that, in the equations for $v_{p}(t)$ and $x_{p}(t)$, we have redefined the origin of time as $t \rightarrow t-t_{n}$. In obtaining the map, it is convenient to work in terms of dimensionless variables and so we define $V_{n}$ $=v_{n} /(\omega l), \delta=\eta / \omega, \epsilon=\varepsilon / l$ and $\phi_{n}=\omega t$. The mapping that describes the dynamics of the model can then be written as

$$
T:\left\{\begin{array}{l}
V_{n+1}=\left|V_{n}-2 \delta-2 \epsilon \sin \left(\phi_{n+1}\right)\right| \\
\phi_{n+1}=\phi_{n}-\frac{1}{\delta} \ln \left[1-\frac{2 \delta}{V_{n}}\right] \bmod 2 \pi
\end{array} .\right.
$$

The second term in the second equation of (1) denotes the dimensionless time that elapses between two collisions.

Let us briefly discuss the modulus function used in Eq. (1). Noting that both walls are fixed, we can immediately see that successive collisions that are allowed in the FUM cannot occur in the SFUM. In the complete model, depending on the velocity and phase, it is possible for the particle, after suffering one collision with the moving wall, to suffer a second (i.e., "successive") collision before exiting the collision area, and it can also have a negative velocity following the first collision. In the simplified model, after a collision with the wall located at $x=0$ nonpositive velocities are forbidden because they are equivalent to the particle traveling beyond the wall. In order to avoid such problems, if after a collision the particle has a negative velocity, we inject it back with the same modulus of velocity. Such a procedure is effected perfectly by use of the modulus function. Note that the velocity of the particle is reversed by the module function only if, after the collision, the particle continues traveling in the negative direction. The modulus function has no effect on the motion of the particle if it moves in the positive direction after the collision. We stress that this approximation is valid only for small values of $\epsilon$.

The phase $\phi_{n+1}$ is defined as a real number only when $V_{n}>2 \delta$. If however $V_{n} \leqslant 2 \delta$, we thus conclude that the particle does not have enough energy for a further collision and therefore comes to rest. The Jacobian of the mapping (1) is written as

$$
J=\left(\begin{array}{cc}
\frac{\partial \phi_{n+1}}{\partial \phi_{n}} & \frac{\partial \phi_{n+1}}{\partial V_{n}} \\
\frac{\partial V_{n+1}}{\partial \phi_{n}} & \frac{\partial V_{n+1}}{\partial V_{n}}
\end{array}\right),
$$

with coefficients given by

$$
\begin{aligned}
\frac{\partial \phi_{n+1}}{\partial \phi_{n}}= & 1, \quad \frac{\partial \phi_{n+1}}{\partial V_{n}}=-\frac{2}{V_{n}^{2}-2 \delta V_{n}}, \\
\frac{\partial V_{n+1}}{\partial \phi_{n}}= & \operatorname{sgn}\left[V_{n}-2 \delta-2 \epsilon \sin \left(\phi_{n+1}\right)\right] \\
\times & {\left[-2 \epsilon \cos \left(\phi_{n+1}\right) \frac{\partial \phi_{n+1}}{\partial \phi_{n}}\right], } \\
\frac{\partial V_{n+1}}{\partial V_{n}}= & \operatorname{sgn}\left[V_{n}-2 \delta-2 \epsilon \sin \left(\phi_{n+1}\right)\right] \\
& \times\left[1-2 \epsilon \cos \left(\phi_{n+1}\right) \frac{\partial \phi_{n+1}}{\partial V_{n}}\right],
\end{aligned}
$$

where the function $\operatorname{sgn}(u)=1$ if $u>0$ and $\operatorname{sgn}(u)=-1$ if $u$ $<0$.

Careful investigation of the determinant of the Jacobian matrix shows that the mapping (1) yields det $J=\operatorname{sgn}\left[V_{n}-2 \delta\right.$ $\left.-2 \epsilon \sin \left(\phi_{n+1}\right)\right]$. This result tells us that it is in principle possible for there to be regions of phase space where area preservation is satisfied. We will show, however, that this result is not applicable throughout the whole of phase space. We begin investigating the behavior in the high energy regime. The first equation of (1) may be rewritten as

$$
\begin{gathered}
V_{1}=V_{0}-2 \epsilon \sin \left(\phi_{1}\right)-2 \delta, \\
V_{2}=V_{0}-2 \epsilon\left[\sin \left(\phi_{1}\right)+\sin \left(\phi_{2}\right)\right]-4 \delta, \\
V_{3}=V_{0}-2 \epsilon\left[\sin \left(\phi_{1}\right)+\sin \left(\phi_{2}\right)+\sin \left(\phi_{3}\right)\right]-6 \delta,
\end{gathered}
$$

and then the general expression as

$$
V_{n}=V_{0}-2 \epsilon \sum_{i=1}^{n} \sin \phi_{i}-2 n \delta .
$$

The analysis of Eq. (3) allows us to conclude that the velocity of the particle decreases linearly as the iteration number $n$ increases. However, even supposing that $\phi$ were uniformly distributed in the interval $\phi \in[0,2 \pi)$ so that on average $2 \epsilon \sum_{i=1}^{m} \sin \phi_{i}=0$, we would still expect the velocity to oscillate according to the $\sin \left(\phi_{n}\right)$ function (an effect seen only for a small interval of $n$ ) with increasing $n$ as time evolves. This is indeed the case, as shown in Fig. 1(a) which plots the calculated velocity as a function of $n$ for $\epsilon=1 \times 10^{-3}, \delta=1$ $\times 10^{-5}$, with the initial condition $V_{0}=1$ and $\phi_{0}=0$. Figure 1(a) shows that the linear decrease in velocity persists over a large range of $n$. The inset of Fig. 1(a) shows in detail the behavior of the velocity $V$ as function of $n$ when the particle passes near a KAM island. Figure 1(b) shows what happens in the phase plane $V \times \phi$. For visual clarity, we have only plotted every fourth point. Thus, for each pair of successive points connected by the dotted line (as a guide to the eye) there are another three that were not plotted. Note that the gap shown in the inset of Fig. 1(a) has the same amplitude $\Delta V$ as that in Fig. 1(b). Proceeding with a linear fit in the regime of high $V$, as shown in Fig. 1(a), we find that the angular coefficient is given by $-1.9990(7) \times 10^{-5}$, as predicted by Eq. (3). 

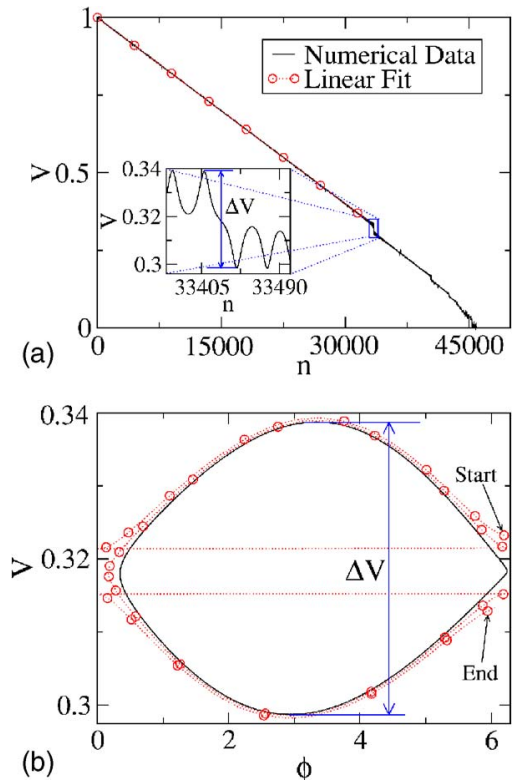

FIG. 1. (Color online) (a) The velocity $V(n)$ for $\epsilon=1 \times 10^{-3}, \delta$ $=1 \times 10^{-5}$ for $V_{0}=1$ and $\phi_{0}=0$. The inset shows in more detail how $V$ varies with $n$ near to the KAM island of order $i=1$. (b) Details of a trajectory passing near to a KAM island, plotting $V(n)$ as a function of the phase $\phi$.

Let us now discuss the behavior of the velocity in the region of the KAM islands. The stable regions for the simplified version of our model are shown in Fig. 2. There is a set of KAM curves, each surrounding an elliptic fixed point that is represented as a dot in Fig. 2. The parameters used in Fig. 2 were $\epsilon=1 \times 10^{-3}$ and $\delta=1 \times 10^{-5}$. For this combination of control parameters, the period-one elliptic fixed points given by $V=2 \delta /[1-\exp (-2 \pi \delta i)]$ and $\phi=\pi$

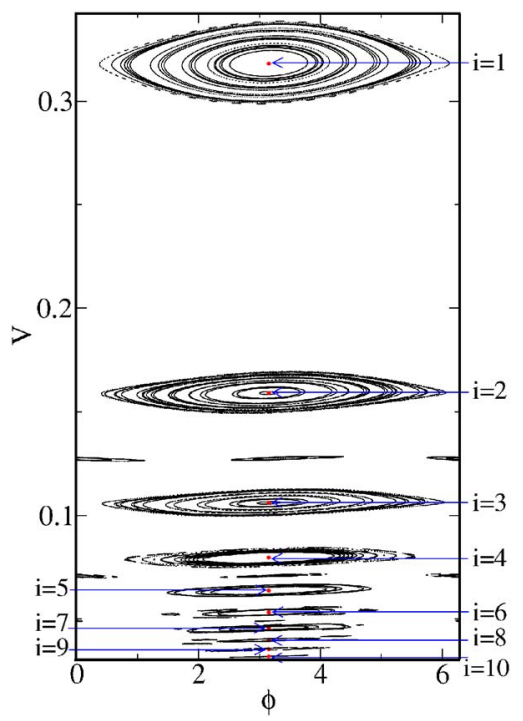

FIG. 2. (Color online) Stable regions in the velocity $V$ vs phase $\phi$ phase space for the dissipative SFUM. The parameters used were $\epsilon=1 \times 10^{-3}$ and $\delta=1 \times 10^{-5}$. The period-one stable fixed points are represented by the dots and classified by the label $i$ ( $i$ $=1,2,3 \ldots 10)$, as shown.
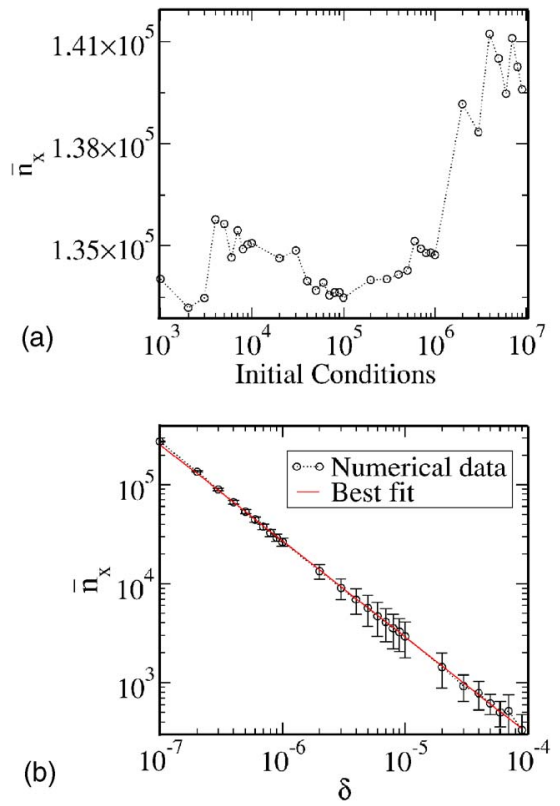

FIG. 3. (Color online) (a) Behavior of the iteration number $\bar{n}_{x}$ as a function of the ensemble of initial conditions for the low energy region using $\delta=2 \times 10^{-7}$ in the simplified version of the dissipative model. (b) The iteration number for the relaxation transient as a function of the parameter $\delta$. The error bars represent the standard deviation for the ensemble of initial conditions used.

$+\arcsin (\delta / \epsilon)$ are stable for $i=1,2,3, \ldots \leqslant 10$ and $\delta / \epsilon \leqslant 1$. Moreover, outside these KAM curves, the particle behaves quite differently. We have shown that, in the regime of high energy, the velocity of the particle decreases linearly as the iteration number increases. It passes around the stable KAM islands [see Fig. 1(b)] and then wanders chaotically (in a chaotic transient) in the low energy domain for a number of iterations that depends on the strength of the drag coefficient. Along the chaotic transient, the particle might momentarily acquire small velocity increases. Once $V_{n} \leqslant 2 \delta$, however, the particle will have insufficient energy to reach the active wall for its next kick, and it therefore comes to rest.

We now characterize this transient for the dissipative SFUM in the low energy domain. To do so, we will evaluate the time evolution $n$ for an ensemble of $m$ different initial conditions in the regime of low energy. We will take as initial conditions different points uniformly distributed in the chaotic sea for the nondissipative case, and then study their asymptotic evolution in the dissipative version. However, as the drag force can cause some modifications to the form of the phase space, it is possible for some regions of the chaotic sea in the nondissipative case to yield periodic or quasiperiodic behavior in the dissipative version. If we find we have taken an initial condition leading to periodic or quasiperiodic behavior in the dissipative model, it is disregarded and a different initial condition is then considered. In order to characterize the deviations around the average value, Fig. 3(a) shows the behavior of the average transient iteration number $\bar{n}_{x}$, i.e., the number of iterations needed to bring the particle to rest, as a function of the number of initial conditions. Figure 3(b) illustrates the behavior of the average transient $\bar{n}_{x}$ as a function of the strength of the drag coefficient. 
The error bars represent the standard deviation of the transient averaged over a set of $m=10^{7}$ different initial conditions [horizontal axis of Fig. 3(a)]. These results allow us to describe the transient as

$$
\bar{n}_{x} \propto \delta^{\mu}
$$

where a power law fit gives us the exponent $\mu=-0.973$ (5) $\approx-1$. It is interesting to observe that Eq. (4) diverges in the limit $\delta \rightarrow 0$. We can still conclude that in this limit of $\delta$ and as a consequence of the divergence of $\bar{n}_{x}$, the dynamics for such a region becomes chaotic. It is also interesting to emphasize that all the invariant spanning curves are now stable in the limit $\delta \rightarrow 0$; a result that, in a sense, establishes the limits on the extent of the chaotic sea.

We now consider why the area-preserving property is not applicable over the whole phase space of the dissipative SFUM. We have shown that, for high energy, the velocity of the particle decreases linearly as the iteration number evolves, necessarily bringing the particle to the region where the chaotic transient arises. As already discussed, however, the particle may assume very low velocities within this regime. The equations defining the map [see Eq. (1)] are restricted (being defined for real numbers) to the range $V_{n}$ $>2 \delta$. If the particle acquires a velocity $V_{n} \leqslant 2 \delta$, the phase $\phi_{n+1}$ is not defined as a real number, corresponding to it no longer having sufficient energy for another collision. As an immediate consequence, the dynamics of the system is over. We note that the condition $V_{n} \leqslant 2 \delta$ breaks down the property of area preservation, because the map is then undefined in this limit of $V_{n}$. Within the stable KAM islands that surround the elliptic fixed points (see Fig. 2), the particle's velocity never falls to $V_{n} \leqslant 2 \delta$. Once within a KAM island, for which the phase $\phi$ is real for all the corresponding velocities, then dissipation is exactly balanced by the energy gained from the periodic forcing, and area preservation applies.

Let us now address this apparent paradox. The interested reader will be able to find a specific example and a more complete discussion in Ref. [30]. First we emphasize that the definition of a dissipative system is not quite so clear as it seems at first sight. One might say that "dissipative" implies that the phase space volume is not conserved under time evolution. Alternatively, one might say that dissipative denotes that friction is present. In our model, however, we have, by construction, a friction force present but, counterintuitively, the determinant of the Jacobian matrix is unity. Why does this result not contradict the statement that there are no attractors in the model studied? The answer is related to the Poincare recurrence theorem. This theorem, which is also a consequence of the Liouville's theorem, states that for a bounded phase space, almost all trajectories eventually return arbitrarily close to where they started. It is interesting to note that this result is true regardless of whether the trajectory under consideration is regular or chaotic. Our results for high energy show convincingly that the particle velocity decreases linearly as the iteration number increases. Eventually the particle enters the corresponding region of the chaotic sea (for the nondissipative case) and we observe that $V \rightarrow 0$. Thus, it is easy to conclude that the time for the next collision $t \rightarrow \infty$, so that the phase space is unbounded. We also comment that in the present dissipative version there is no mechanism for accelerating the particle to high energy (say higher than $V_{c} \cong 2 \sqrt{\epsilon / 0.97 \ldots}$, see Ref. [4]). Thus the Poincaré recurrence argument is not satisfied.

Finally, we point out that the coexistence of conservative and dissipative behavior has also been observed in a laser [31], where it was attributed to the occurrence of a symmetry-breaking bifurcation, leading to the appearance of a structurally stable homoclinic cycle.

\section{CONCLUSIONS}

Although one might have expected dissipation to destroy all the mixed phase space structure present in the nondissipative Fermi-Ulam accelerator model, we have found that this is not the case. Dissipation does indeed destroy the invariant spanning curves and chaotic sea characterizing the nondissipative model. Yet its KAM islands can survive the perturbation. Counterintuitively, therefore, regions of phase space exist where the dissipative SFUM exhibits the property of area-preservation.

\section{ACKNOWLEDGMENTS}

E.D.L. is grateful to CNPq and FAPESP, Brazilian agencies; P.V.E.McC. gratefully acknowledges support from the Engineering and Physical Sciences Research Council (UK).
[1] E. Fermi, Phys. Rev. 75, 1169 (1949).

[2] A. J. Lichtenberg and M. A. Lieberman, Regular and Chaotic Dynamics, Appl. Math. Sci. 38 (Springer Verlag, New York, 1992).

[3] M. A. Lieberman and A. J. Lichtenberg, Phys. Rev. A 5, 1852 (1972).

[4] E. D. Leonel, J. K. L. da Silva, and S. O. Kamphorst, Physica A 331, 435 (2004).

[5] E. D. Leonel, P. V. E. McClintock, and J. K. da Silva, Phys. Rev. Lett. 93, 014101 (2004).

[6] D. G. Ladeira and J. K. L. da Silva, Phys. Rev. E 73, 026201
(2006)

[7] L. D. Pustylnikov, Trudy Moskov. Mat. Obsc. 34, 1 (1977).

[8] A. J. Lichtenberg, M. A. Lieberman, and R. H. Cohen, Physica D 1, 291 (1980).

[9] E. D. Leonel and P. V. E. McClintock, J. Phys. A 38, 823 (2005).

[10] G. Karner, J. Stat. Phys. 77, 867 (1994).

[11] S. T. Dembinski, A. J. Makowski, and P. Peplowski, Phys. Rev. Lett. 70, 1093 (1993).

[12] J. V. José and R. Cordery, Phys. Rev. Lett. 56, 290 (1986).

[13] P. Seba, Phys. Rev. A 41, 2306 (1990). 
[14] S. R. Jain, Phys. Rev. Lett. 70, 3553 (1993).

[15] A. Mehta and J. M. Luck, Phys. Rev. Lett. 65, 393 (1990).

[16] J. P. Holmes, J. Sound Vib. 84, 173 (1982).

[17] J. M. Luck and A. Mehta, Phys. Rev. E 48, 3988 (1993).

[18] K. Y. Tsang and M. A. Lieberman, Physica D 21, 401 (1986).

[19] M. A. Lieberman and K. Y. Tsang, Phys. Rev. Lett. 55, 908 (1985).

[20] See Ref. [3], Chap. 8, Sec. 8.1a.

[21] R. M. Everson, Physica D 19, 355 (1986).

[22] E. D. Leonel and P. V. E. McClintock, J. Phys. A 38, L425 (2005).

[23] M. A. Naylor, P. Sánchez, and M. R. Swift, Phys. Rev. E 66, 057201 (2002).
[24] G. A. Luna-Acosta, Phys. Rev. A 42, 7155 (1990).

[25] Z. J. Kowalik, M. Franaszek, and P. Pieranski, Phys. Rev. A 37, 4016 (1988)

[26] S. Warr, W. Cooke, R. C. Ball, and J. M. Huntley, Physica A 231, 551 (1996).

[27] S. Warr and J. M. Huntley, Phys. Rev. E 52, 5596 (1995).

[28] M. Robnik and M. V. Berry, J. Phys. A 18, 1361 (1985).

[29] K. Y. Tsang and K. L. Ngai, Phys. Rev. E 56, R17 (1997).

[30] G. J. Sussman, J. Wisdom, and M. E. Mayer, Structure and Interpretation of Classical Mechanics (MIT Press, Cambridge, 2001).

[31] A. Politi, G. L. Oppo, and R. Badii, Phys. Rev. A 33, 4055 (1986). 